\title{
Miniaturized Dielectric Resonator Antenna Array for GNSS Applications
}

\author{
S. Caizzone, G. Buchner, and W. Elmarissi \\ Institute of Communications and Navigation, German Aerospace Center (DLR), 82234 Wessling, Germany \\ Correspondence should be addressed to S. Caizzone; stefano.caizzone@dlr.de
}

Received 2 May 2016; Revised 6 June 2016; Accepted 13 June 2016

Academic Editor: Yingsong Li

Copyright ( 92016 S. Caizzone et al. This is an open access article distributed under the Creative Commons Attribution License, which permits unrestricted use, distribution, and reproduction in any medium, provided the original work is properly cited.

\begin{abstract}
The increase in global navigation satellite systems (GNSS) availability and services is fostering a new wave of applications related to satellite navigation. Such increase is also followed by more and more threats, aiming at signal disruption. In order to fully exploit the potentialities of precise and reliable navigation, being able at the same time to counteract threats such as interference, jamming, and spoofing, smart antenna systems are being investigated worldwide, with the requirements of multiband operation and compactness. In order to answer such need, the present work proposes a miniaturized dielectric resonator antenna (DRA) $2 \times 2$ array able to operate at E5/L5, L2, and E6 bands, with an overall footprint of only $3.5^{\prime \prime}(89 \mathrm{~mm})$.
\end{abstract}

\section{Introduction}

The increase in GNSS constellations and services alongside with the GPS system, as, for instance, the forthcoming Galileo system, enables a plethora of novel applications concerning the precise positioning of people, vehicles, and goods. Such spreading of the relevance of satellite navigation in different applications also causes more and more interest in malicious attacks, for instance, through jamming or spoofing, to deny the service [1] or even try to deceive the navigation functionality [2].

In order to fully exploit the offered possibilities and at the same time counteract possible threats, high-performance systems, able to steer the antenna pattern and hence place nulls against interferences and maxima at the direction of arrival (DOA) of signals, are being developed all over the world (see, e.g., [3]). These new systems might be particularly useful in mobile applications, such as aircraft or vehicles, where precise localization can play an important role. However, the canonical dimensions of the antennas and of receivers do not fit with the requirements of low profile and low weight, typical of mobile applications: in the aeronautic sector, for instance, the tolerable size is regulated by ARINC standard dimensions [4]. There is hence a need for miniaturization both of the single radiator and of the overall antenna array, to enable real-life implementation of the developed systems.

Moreover, benefits can be easily foreseen if antennas and receivers are able to process at the same time signals coming from multiple bands, with increases in precision, availability, and robustness [5].

A few different works are already available regarding robust and reliable satellite navigation receivers: in [3], a single band $2 \times 2$ array is shown, together with a complete receiver, demonstrating the smart antenna capability for interference suppression. Proper algorithms for adaptive array processing for robust navigation are, for instance, reported in [6-8]. Concerning the geometry of the antenna array, different nonplanar geometries are studied in [9-11], showing optimal array performance at low-elevation, but having relatively large dimensions.

On the other hand, in [12], miniaturization of the system (and hence of the antenna array) for use at L1 band is pursued, achieving a size of $10 \times 10 \mathrm{~cm}^{2}$ (diameter $141 \mathrm{~mm}$ ). Moreover, a dual-band system at L1 and L2 bands is reported in [13], having however an overall diameter of $165 \mathrm{~mm}$, hence still too big, for instance for, aeronautical requirements.

The need for further work on antenna miniaturization for GNSS arrays is therefore evident. Antenna miniaturization 
can be pursued by means of different techniques (see [14] for a broad review): the geometry of the antenna can be changed by using meandering, effectively enlarging the current path, and hence lowering the resonance frequency [15]; however, this technique usually strongly degrades the polarization purity of circularly polarized antennas, hence not being suited for precise GNSS applications. Material loading is also possible, by using materials with high dielectric or magnetic constants, which effectively provide a wave slowdown and hence antenna miniaturization, normally at the cost of reduced bandwidth. At L-band, there are a few low loss materials with high dielectric constant (DK), while materials with high magnetic constants usually are more lossy at theses frequencies and find therefore little application at L-band. Finally, metamaterials, as well as electromagnetic band gap (EBG) materials [16], can be used to pursue miniaturization: they are however usually bulky and would increase relevantly the cost of the antenna.

Among the above techniques, material loading with high DK materials seems particularly promising in GNSS applications, because it allows for strong miniaturization of structures, without affecting polarization purity and without the need for additional layers, as instead is the case for metamaterials.

In this work, therefore, a bulk glass ceramic material with a DK of $\sim 30$ will be used: this will enable a single antenna diameter of only $30.5 \mathrm{~mm}$, which will then allow obtaining the very compact $2 \times 2$ array footprint of $89 \mathrm{~mm}$ (3.5 inches), remarkably smaller than the ones previously reported and fulfilling compactness requirements.

The single antennas are dielectric resonator antennas (DRAs) and are designed for operation at E5/L2/E6 bands. Special measures will be shown that minimize mutual coupling between the very close radiators.

The paper is therefore divided as follows: Section 2 shows the single antenna design, while array design is explained in Section 3. Measurement results are reported in Section 4. Finally, conclusions are drawn in Section 5.

\section{Single Antenna Design}

2.1. Modality of Operation. Dielectric resonators (DRs) [17, 18] have been thoroughly analyzed in the last decades and are now used for various applications, such as microwave filters and oscillators. They have the characteristic property of having very few metal parts and hence exhibit quite low losses even at very high frequency. Thanks to such feature, their use as antennas is now becoming popular, because low losses allow high radiation efficiency. Their behaviour is based on the resonant mode(s) of a dielectric cavity, established by means of a feeding element. Such mode will however not be totally confined into the dielectric material but will also leak out, leading to radiation.

By using dielectric resonator antennas (DRAs) [18] for miniaturized arrays, two additional advantages are available: on one hand, they can make use of very high DK dielectric materials, hence enabling strong miniaturization, while exhibiting a relatively broader bandwidth than microstrip antennas [19]. On the other hand, they are not planar, but volumetric structures and hence give designers additional degrees of freedom during the design phase.

In the present work, a bulk glass ceramic material from Schott AG (Schott GHz 33, with DK of about 33) has been taken into consideration for the DRA. The high DK value will allow strong miniaturization of the single antenna and therefore permit obtaining a more compact array. The material has superior properties in terms of purity (and hence has minimal DK tolerances) and temperature dependence. The antenna has a cylindrical shape and is placed on a $3.5^{\prime \prime}$ metallic plate (Figure 1(a)).

Two metallic strips, conformal to the DRA surface and soldered to the inner conductor of SMA connectors, act as feeds for the DRA, exciting orthogonal linear polarized fields (namely, mode $\mathrm{HEM}_{11 \delta}$ along $x$ - or $y$-axis, resp.). The height of the metallic strips can be properly chosen for optimal matching, hence providing an additional tuning parameter. RHCP polarization is then obtained with the use of a commercial $90^{\circ}$ hybrid combiner (ZX10Q-2-19-S+ from Mini-Circuits, exhibiting good behaviour in the whole Lband), connected to the two SMA connectors, that is, to the feeds of the two orthogonal linear fields. Finally, the upper surface of the cylinder is fully covered by copper ("copper cap," Figure 1(a)): this helps [20] in obtaining even further miniaturization.

2.2. Analysis of the DRA. In order to fully master the behaviour of the antenna and to identify the tuning parameters for optimal matching, a series of numerical simulations have been performed with an electromagnetic (e.m.) solver, ANSYS HFSS, showing the effect of variable geometrical parameters on the antenna characteristics. In particular, the radius $(r)$ and height $(h)$ of the DRA cylinder and the length of the copper strips $\left(l_{\mathrm{cs}}\right)$ and the radius of the copper cap $\left(r_{\mathrm{cc}}\right)$ have been taken into account. The results are shown in Figures 2 and 3.

The radius of the cylinder and the radius of the copper cap are mostly effective on the resonance frequency, while the length of the copper strip and the height of the cylinder act mostly on the impedance values (i.e., sharpening or flattening the resonant peaks), hence turning useful in the search for optimal matching once the correct resonance frequency has been found.

The copper cap actually perturbates the fields of mode $\mathrm{HEM}_{11 \delta}$ (excited through probe feeding), by imposing a metal boundary condition (i.e., the need for nonzero $E$ field components directed only normally to the surface) at the upper face of the DRA. Such perturbation however does not change the essential radiation characteristics of the antenna [21], which remains, as for the pure $\mathrm{HEM}_{11 \delta}$ mode, substantially pointing towards zenith (i.e., $z$-axis in Figure 1), as requested for GNSS antennas.

Nevertheless, the presence of the cap produces a shift of the resonance towards lower frequencies, hence providing an easy, yet powerful, way for further miniaturization.

2.3. Final Design for the Single Antenna. Using the precious information of the former paragraph, it is possible to optimally match the antenna (in standalone configuration): best 


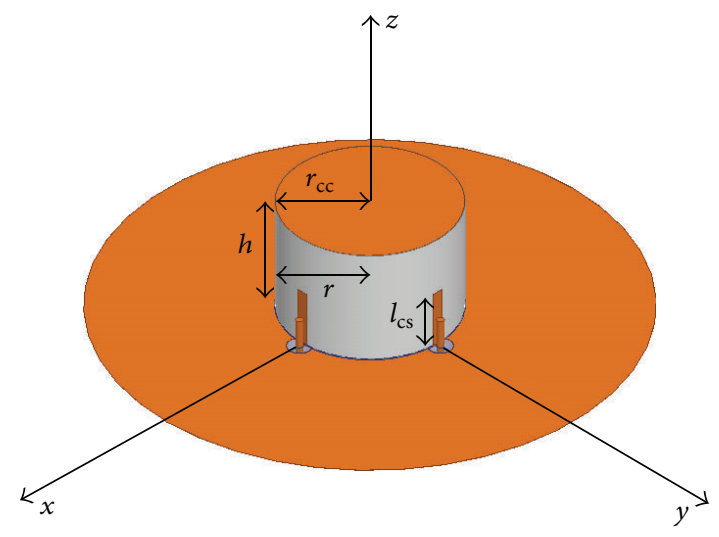

(a)

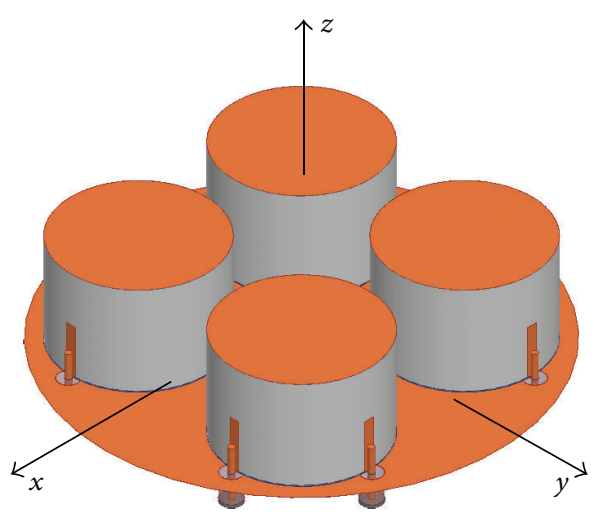

(b)

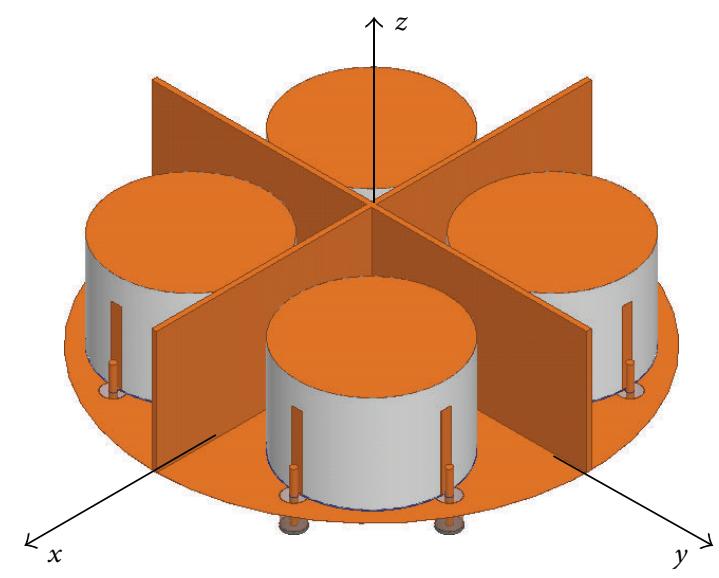

(c)

FIGURE 1: 3D view of the single DRA (a) and $2 \times 2$ DRA array, either without (b) or with metallic fence (c) on a circular ground plane (diameter: $\left.3.5^{\prime \prime}\right)$.

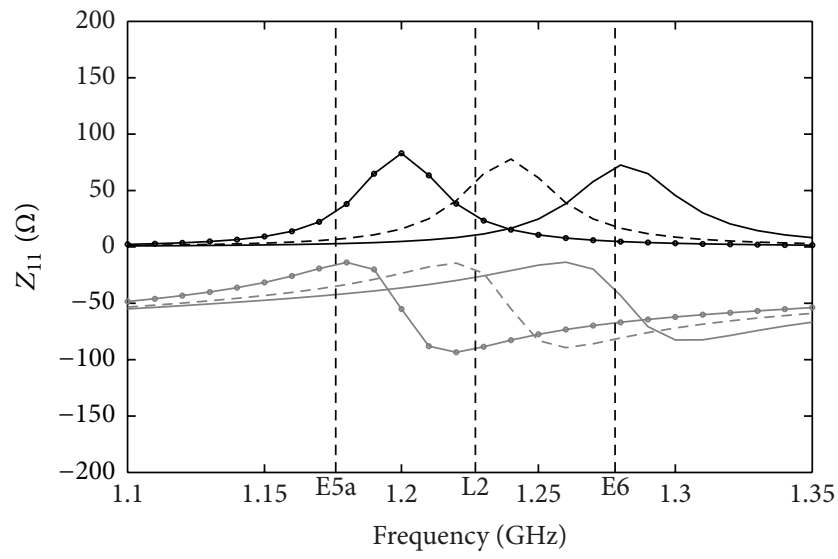

$$
\begin{array}{ll}
-R_{11} r=14.75 \mathrm{~mm} & -X_{11} r=14.75 \mathrm{~mm} \\
---R_{11} r=15.25 \mathrm{~mm} & --X_{11} r=15.25 \mathrm{~mm} \\
--R_{11} r=15.75 \mathrm{~mm} & -X_{11} r=15.75 \mathrm{~mm}
\end{array}
$$

(a)

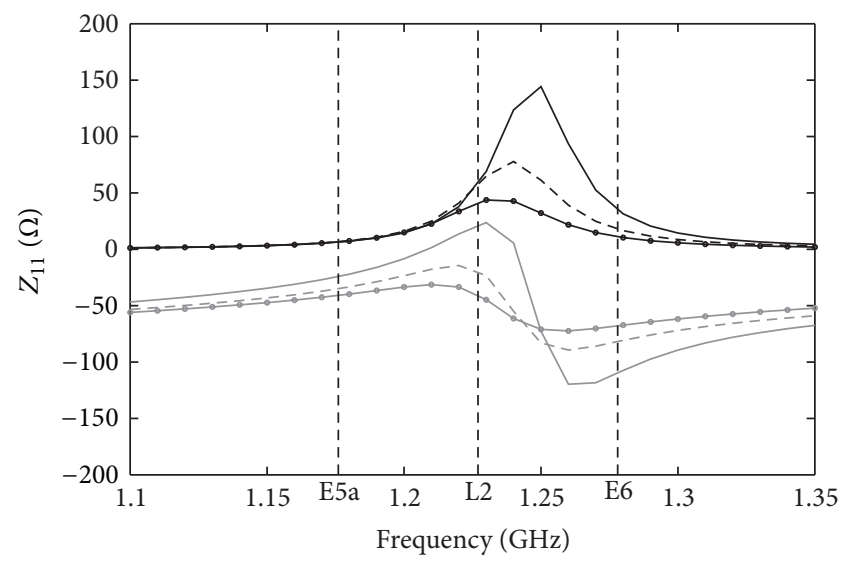

$$
\begin{array}{ll}
-R_{11} h=15 \mathrm{~mm} & -X_{11} h=15 \mathrm{~mm} \\
---R_{11} h=20 \mathrm{~mm} & --X_{11} h=20 \mathrm{~mm} \\
\text { - } R_{11} h=25 \mathrm{~mm} & \text { - } X_{11} h=25 \mathrm{~mm}
\end{array}
$$

(b)

FIGURE 2: $Z_{11}$ of the single DRA with varying parameters: (a) radius of the DRA cylinder; (b) height of the DRA cylinder. 


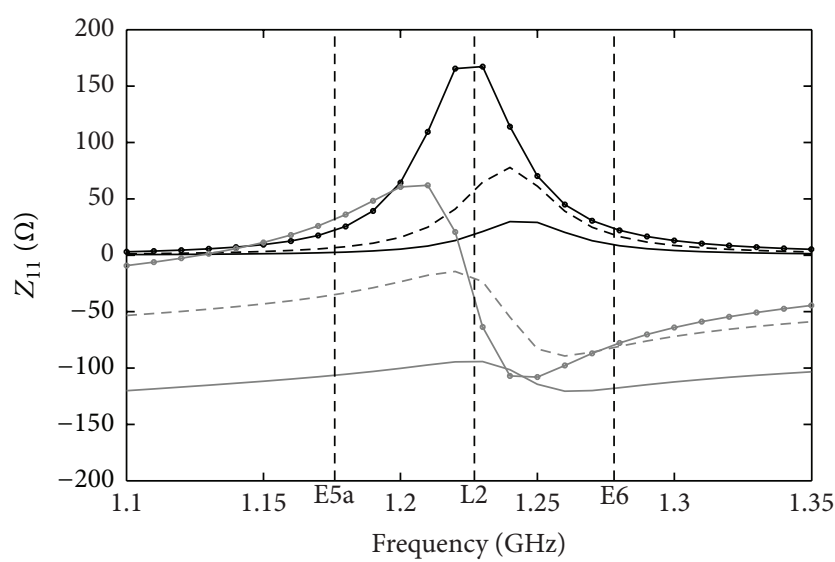

$$
\begin{array}{ll}
-R_{11} l_{\mathrm{cs}}=5 \mathrm{~mm} & -X_{11} l_{\mathrm{cs}}=5 \mathrm{~mm} \\
--R_{11} l_{\mathrm{cs}}=10 \mathrm{~mm} & --X_{11} l_{\mathrm{cs}}=10 \mathrm{~mm} \\
\longrightarrow R_{11} l_{\mathrm{cs}}=15 \mathrm{~mm} & -X_{11} l_{\mathrm{cs}}=15 \mathrm{~mm}
\end{array}
$$

(a)

FIGURE 3: $Z_{11}$ of the single DRA with varying parameters: (a) length of the copper strip; (b) radius of the copper cap.

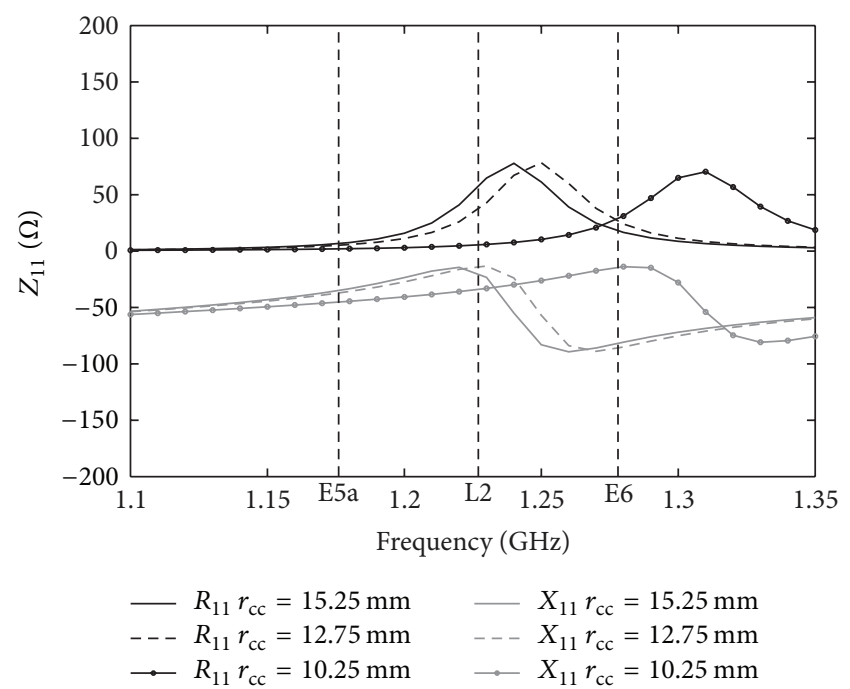

(b)

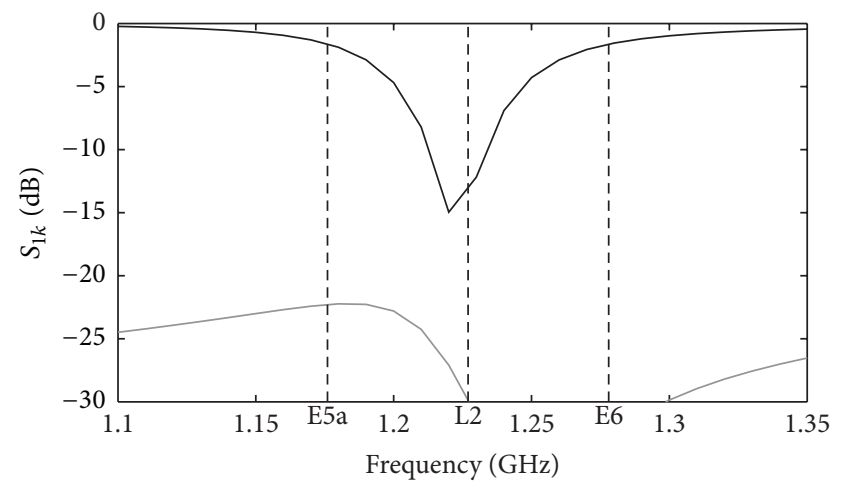

$$
-S_{11}
$$

FIGURE 4: $S_{11}$ and $S_{12}$ of the single DRA on a circular ground plane (diameter: $3.5^{\prime \prime}$ ).

performance is found when the single antenna element has a diameter of $30.5 \mathrm{~mm}$, a height of $20 \mathrm{~mm}$, a length of the copper strips of $10 \mathrm{~mm}$, and a radius of the copper cap of $15.25 \mathrm{~mm}$ (Figure 4).

In this case, the antenna achieves the realized gain depicted in Figure 5. As it can be observed, the single antenna has optimal performance both for gain and for matching at L2 band, that is, in the middle of the frequency range covering also E5 and E6 bands. At $f=1228 \mathrm{MHz}$, the simulated realized gain towards zenith is $4.5 \mathrm{dBic}$, while it is higher than $-3 \mathrm{dBic}$ also at E5a and E6 bands.

\section{Array Design}

The single antenna shown in the previous section has been used as "building block" for the $2 \times 2$ array. Thanks to

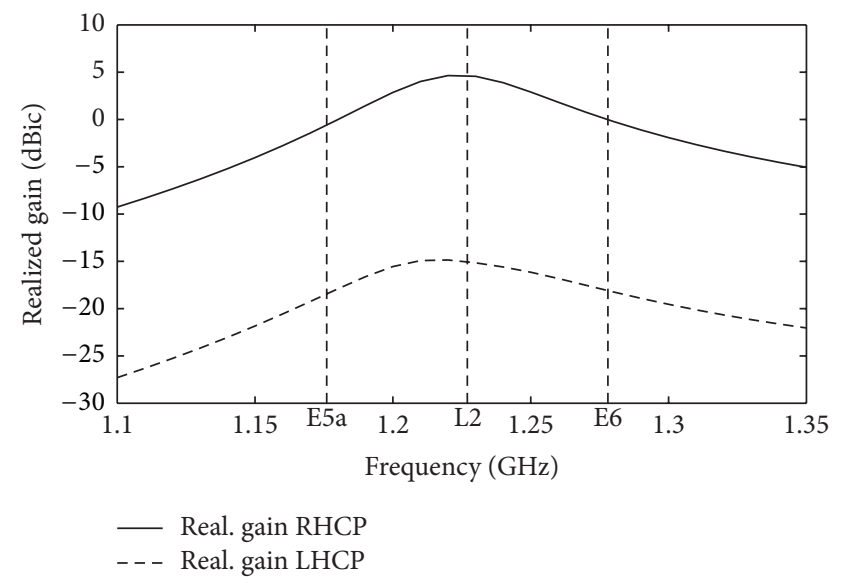

FIGURE 5: Boresight realized gain of the single DRA on a circular ground plane (diameter: $3.5^{\prime \prime}$ ).

the reduced dimension of the single antenna, very small mutual distances between neighbouring elements are achievable, hence allowing obtaining the required very compact footprint. The antennas have been positioned following a sequentially rotated geometry, in order to help in improving polarization purity of the overall array. A $3 \mathrm{D}$ view of the array is shown in Figure 1(b). The array footprint size is $89 \mathrm{~mm}$ and the distance between antennas is $d=37 \mathrm{~mm}$. Results for such configuration will be shown in Section 3.1. Metallic fences have later been placed between the antennas, to limit the mutual coupling effects, due to the very close vicinity of antennas $(d=37 \mathrm{~mm} \approx \lambda / 7$ at E5 band), as discussed in Section 3.2. Finally, an absorber substrate has been added on top of the fence edges to minimize spurious radiation as shown in Section 3.3. 


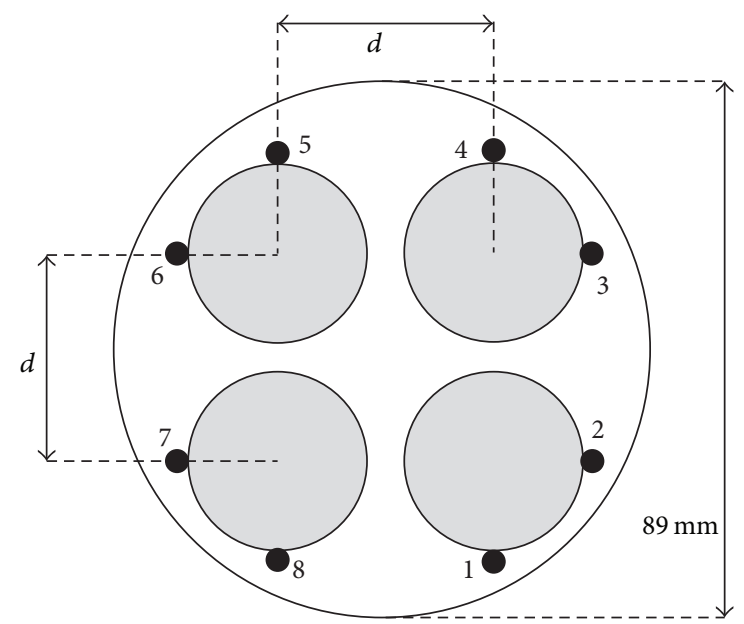

FIGURE 6: Schematic view of the array: each antenna has two ports (exciting two orthogonally polarized fields).

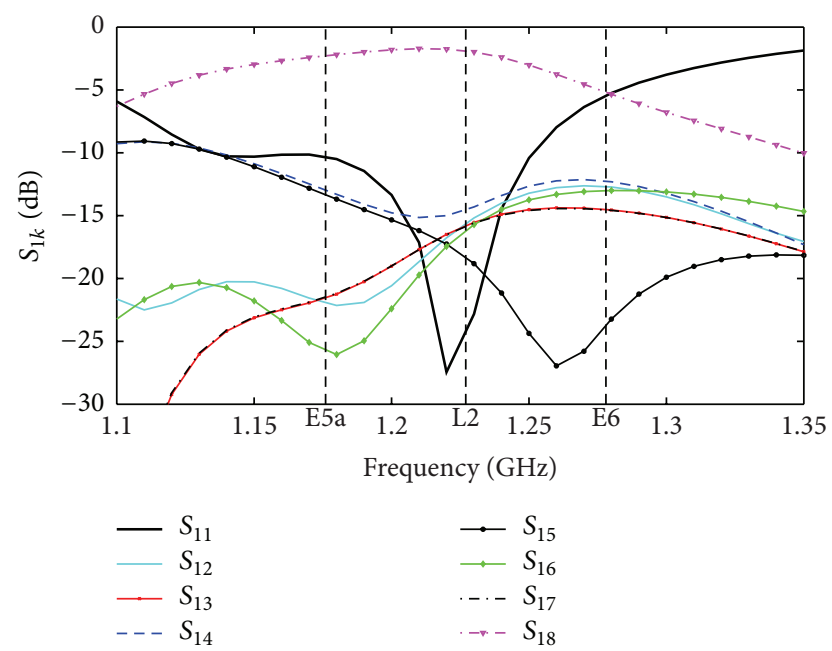

FIgURE 7: $S_{1 k}$ parameters of the DRA array without fences.

3.1. Miniaturized $2 \times 2$ Array. When the antennas are placed in array configuration (sequentially rotated to improve polarization purity), strong mutual coupling is expected between them, due to the very close vicinity.

As a matter of fact, such mutual coupling can be observed in the $S$-parameters for port 1 (each antenna has two ports, representing the two feeding probes. The port numbering can be seen in Figure 6) in Figure 7: for instance, $S_{18} \sim-2 \mathrm{~dB}$ at $f=1.228 \mathrm{GHz}$, implying that more than half of the power of port 1 is coupled to port 8 .

This situation is detrimental, because it strongly reduces both the gain of the antenna (as power is coupled with other ports instead of being radiated/received) and degrades the independence of the signals coming out of each antenna (since the signal will now contain information of multiple ports due to coupling). Moreover, the maximum gain of the embedded antenna decreases and is shifted in frequency (Figure 8).

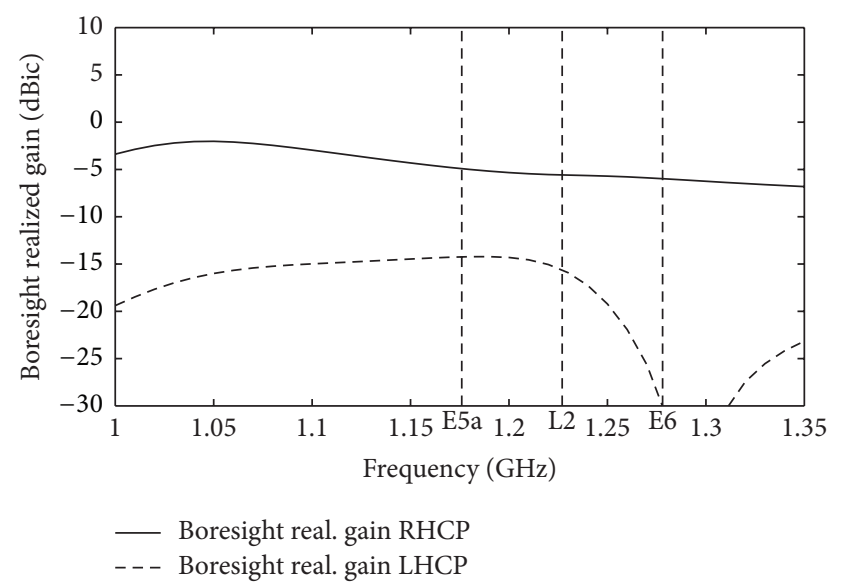

FIGURE 8: Boresight realized gain of the $2 \times 2$ array of DRAs without fence for the embedded case (only antenna $1 \mathrm{fed}$ ).

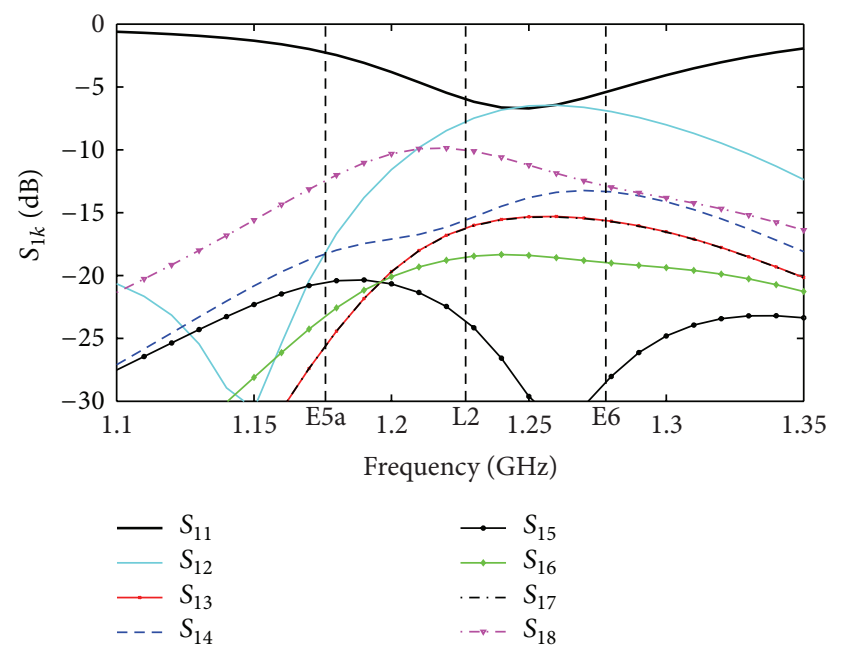

FIGURE 9: $S_{1 k}$ parameters of the optimized DRA array.

A possible solution to the problem is shown in the next subsections.

3.2. Miniaturized $2 \times 2$ Array with Metallic Fences. In order to limit the crosstalk between the different ports of the array, metallic fences (Figure 1(c)) can be used in-between them [22, 23].

The positive effect of fences in reducing mutual coupling can be clearly observed in Figure 9, where $S$-parameters for port 1 are shown: all mutual $S$-parameters are now below $-10 \mathrm{~dB}$, apart from $S_{12}$, which is $\sim-8 \mathrm{~dB}$ at L2 frequency. $\left(S_{12}\right.$ expresses the power flow between the two probes of the same antenna and therefore does not benefit from the addition of the metallic fences. Actually, the fence has even a detrimental effect for $S_{12}$, as it contributes to coupling signal from port 1 to port 2 by mirroring radiated power back towards the antenna itself.)

A substantial decrease in coupling is therefore achieved, which also allows for maximum embedded gain in the bands of interest, as shown in Figure 10. Due to the residual 


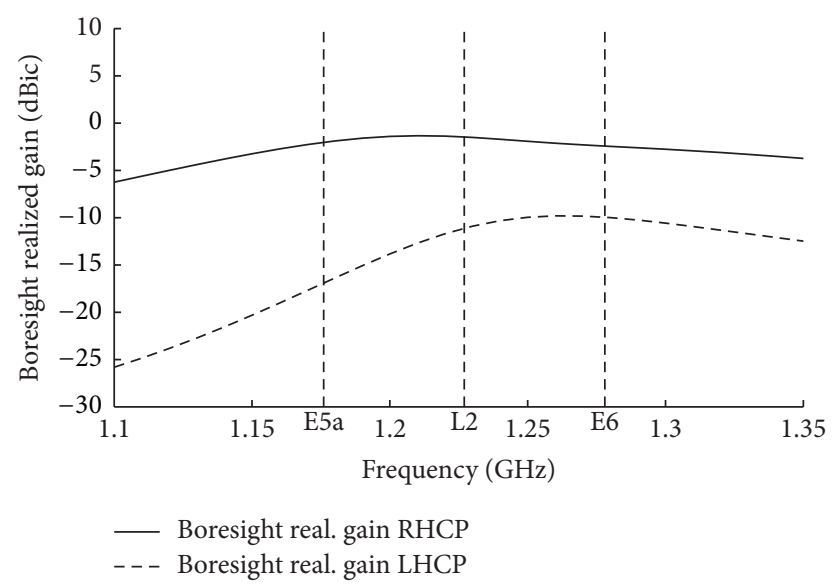

FIGURE 10: Boresight realized gain of the $2 \times 2$ array of DRAs with fence for the embedded case (only antenna $1 \mathrm{fed}$ ).

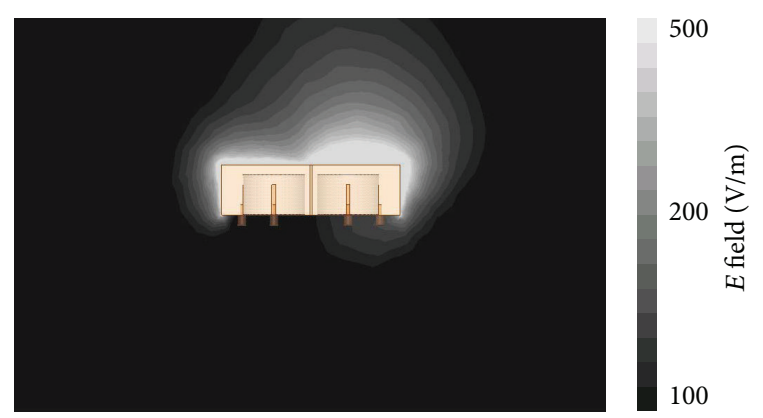

FIGURE 11: $E$-field in the $x z$ plane (vertical plane passing through the fence) for the $2 \times 2$ array of DRAs with fences when one antenna (on the right of the image) is fed. Strong radiation from the fence, for example, on the left side, is clearly visible.

coupling, the gain is however lower than for the standalone configuration. Moreover, high cross polarization is obtained.

The high cross polarization can be ascribed mostly to the spurious radiation caused by the currents flowing on the upper edges of the metallic fence, whose radiation then interferes with the fields originated from the antenna itself (Figure 11). The metallic fences effectively behave as a near field scatterer and hence diminish the polarization purity of the antenna. In order to limit such effect, an absorber can be used, as shown in the next subsection.

3.3. Miniaturized $2 \times 2$ Array with Metallic Fences and Absorber. In order to limit the spurious radiation due to the fence edges, an absorber material can be placed on top of the fence, with the effect of strongly attenuating the currents radiating from the fence edges.

A flexible rubber based microwave absorber from ARC (UD-11091-7), having a thickness of $5.08 \mathrm{~mm}$, has been used (Figure 12). The absorber protrudes vertically $5 \mathrm{~mm}$ from the upper edge of the fences in order to minimize the additional vertical height of the antenna. On the other hand, the depth of the absorber from the fence edges $\left(d_{\mathrm{abs}}\right.$ in Figure 12) shall take into account two contrasting requirements: on one

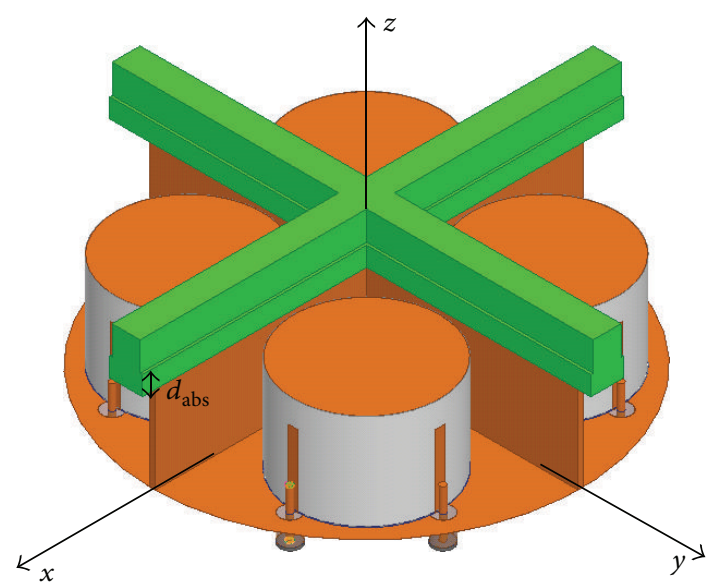

FIGURE 12: 3D view of the $2 \times 2$ DRA array with fences and absorber mounted on their upper edges.

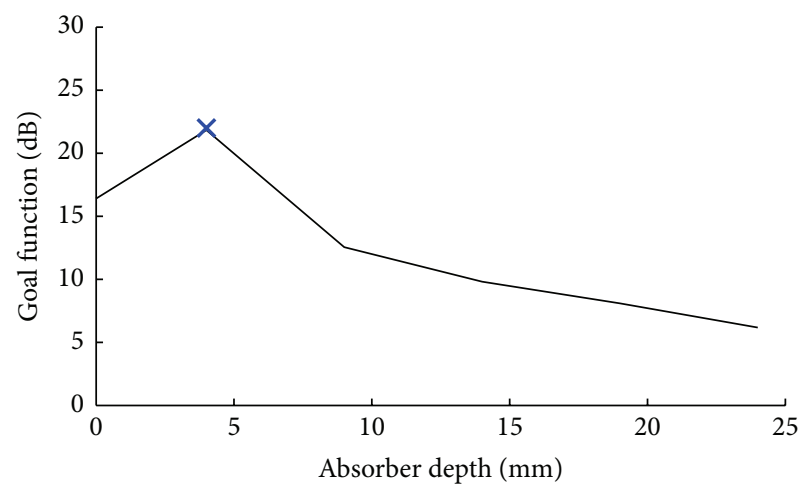

FIGURE 13: Goal function versus absorber depth. The best solution is indicated with the cross $\left(w_{1}=0.5, w_{2}=0.5\right)$.

hand, having an absorber on the whole lateral face of the fence would help in minimizing also the field reflected from the fence and coupled back into the DRA, hence further improving polarization purity; on the other hand, it would also lower the achievable gain, due to the higher energy dissipation in the near field of the antenna.

An optimal result can be found by maximizing the goal function

$$
\text { gf }=w_{1} G_{R, f_{0}}+w_{2} \mathrm{XPD}_{f_{0}},
$$

where $w_{1}$ and $w_{2}$ are weigthing coefficients and $G_{R, f_{0}}$ is the realized gain at boresight at the center frequency $f_{0}=$ $1200 \mathrm{MHz}$ and $\mathrm{XPD}_{f_{0}}$ is the cross polarization discrimination in boresight direction at $f_{0}$.

An optimal result is found (blue cross in Figure 13) when the absorber depth is $5 \mathrm{~mm}$ from the edge (hence implying a total height of the absorber of $10 \mathrm{~mm}$ ): for such case, the RHCP gain at $f_{0}$ is $-1.8 \mathrm{dBic}$ and the XPD $=20 \mathrm{~dB}$ (Figure 14), hence substantially improved with respect to the case with no absorber.

It is worth noticing how the gain, though having a lower peak value, has a broader $3 \mathrm{~dB}$ bandwidth in the array configurations, both with and without absorber, with respect 


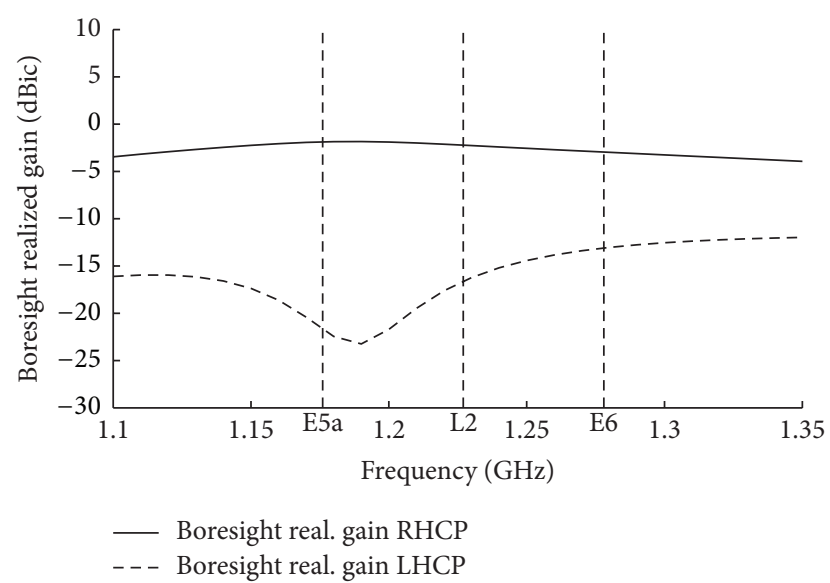

FIGURE 14: Boresight realized gain of the $2 \times 2$ array of DRAs with fence and absorber for the embedded case (only antenna $1 \mathrm{fed}$ ).

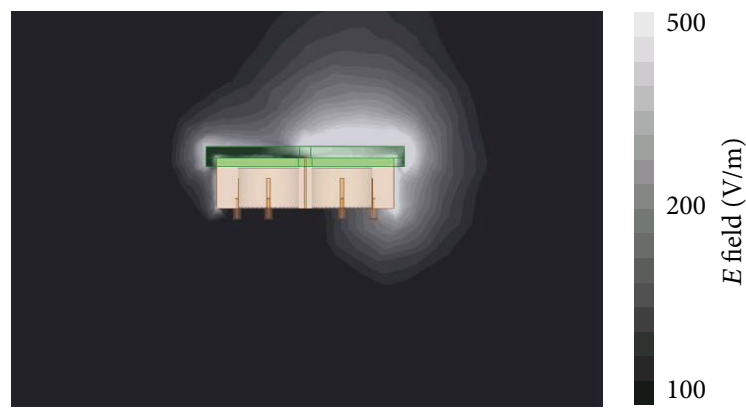

FIGURE 15: $E$-field in the $x z$ plane (vertical plane passing through the fence) for the $2 \times 2$ array of DRAs with fences and absorber. Only one antenna is fed. A reduced radiation from the fence is now visible.

to the single antenna configuration (see, e.g., Figure 20 and Figure 17): this is however a favourable property for our application, as it allows satellite signals received at all bands to have similar power levels, due to the similar antenna gain. Relatively low gain levels are however not too problematic for GNSS applications, as long as the signals can properly be tracked, as is, for instance, the case in mobile phone GNSS antennas, having gains also $15 \mathrm{~dB}$ smaller than high-level antennas, due to their extremely small size [24].

In our case, it is therefore more useful to have multiple antennas closely packed together with lower gain, but providing the possibility for beamsteering and nulling, than a single antenna with fixed pattern and higher gain.

The field radiated by the fence edge is moreover substantially diminished (Figure 15), actively contributing to a better polarization purity. The manufacturing and measurement of the proposed arrays are reported in next section.

\section{Measurement}

The antennas were manufactured in house. In order to enable an easier manufacturing, the copper strips and cap have been obtained through adhesive copper sheet glued to the surface of the glass ceramic cylinder. The DRA has

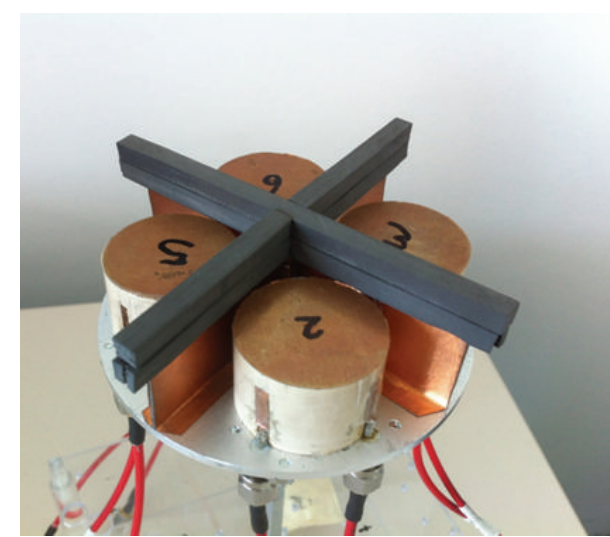

(a)

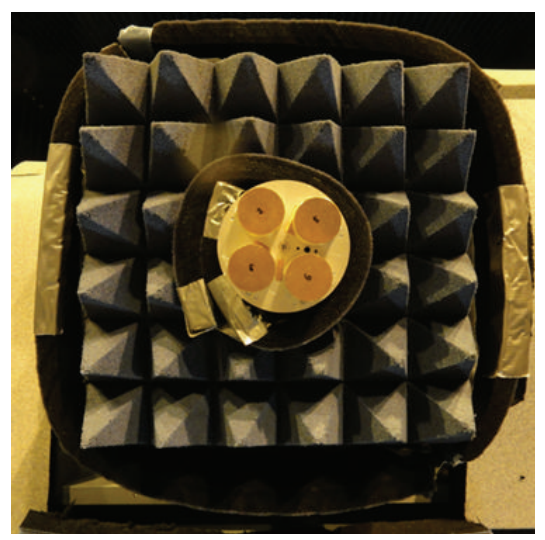

(b)

FIGURE 16: Manufactured array: (a) array with metallic fences and absorber; (b) array without metallic fences, mounted on the movable support of the antenna measurement facility.

then been fixed on the ground plane by means of an RF adhesive tape and the copper strips have been soldered to the inner conductors of SMA connectors. Both the antenna and the arrays (without fences or with fences and absorber) were measured in a near field antenna measurement facility (Satimo StarLab) (Figure 16). External $90^{\circ}$ hybrids are used in field measurement to excite circular polarization, that is, to impose a $90^{\circ}$ shift between the two orthogonal ports of the antenna.

4.1. Single Antenna. The single antenna was measured in the center of a metallic plate with diameter of 3.5 inches (i.e., the same plane size used for the array). The results are shown in Figures 17 and 18, where the measured gain at boresight versus frequency and the $S$-parameters are reported.

The measured $S_{11}$ is slightly worse than the simulated one, most probably due to the nonperfect manufacturing of the strips: improvements are expected if the feeding parts are directly printed on the DRA instead of manually attaching them. The slight mismatch contributes also to a slight degradation of maximum gain, but with an improvement of the bandwidth. Nevertheless, a maximum gain of $3.9 \mathrm{dBic}$ at L2 band is found, quite close to the simulated results. 


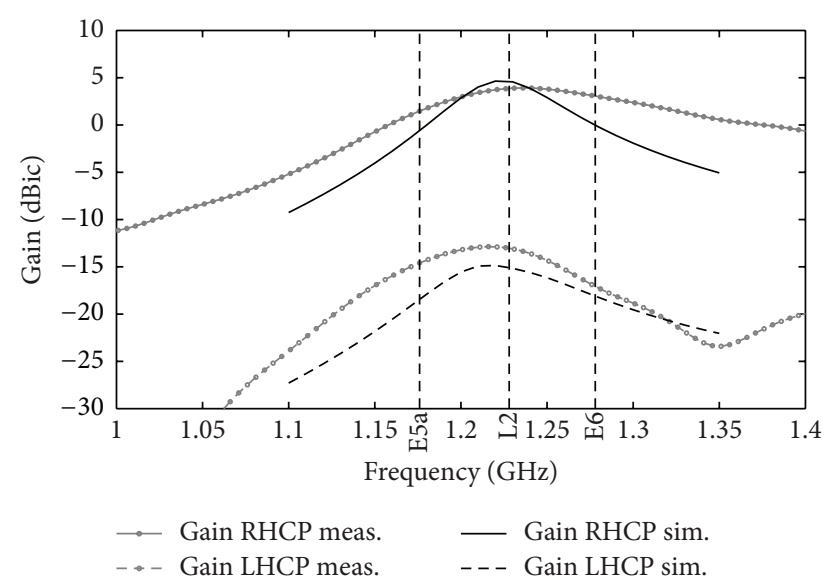

FIGURE 17: Boresight realized gain of the single DRA versus frequency.

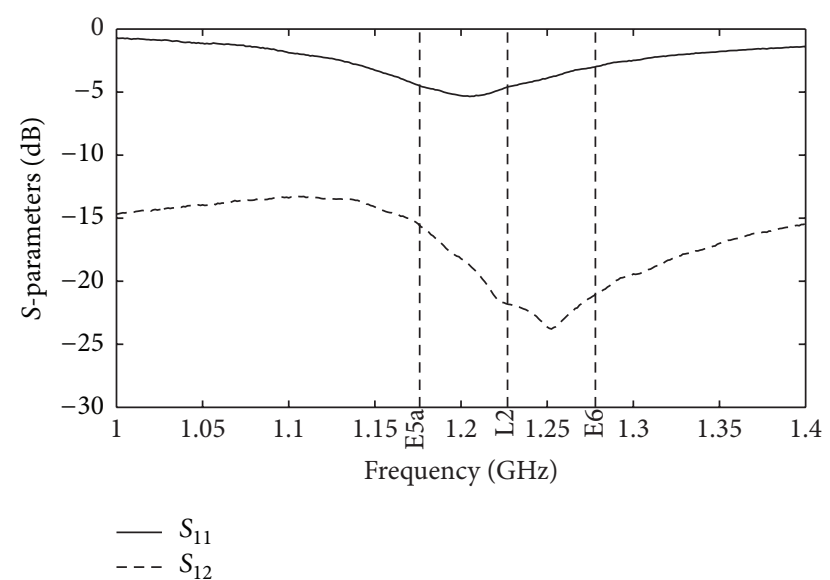

FIGURE 18: Measured S-parameters of the single DRA versus frequency.

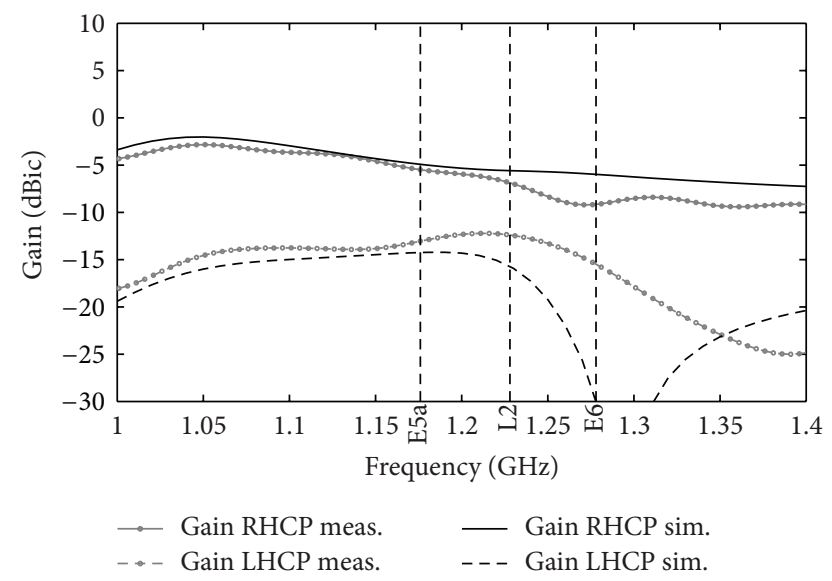

FIGURE 19: Boresight realized gain of the $2 \times 2$ DRA array versus frequency for the embedded case (one antenna fed).

4.2. Miniaturized $2 \times 2$ Array. The miniaturized $2 \times 2$ array without fences has then been measured, with gain results as shown in Figure 19. Sequential rotation of the

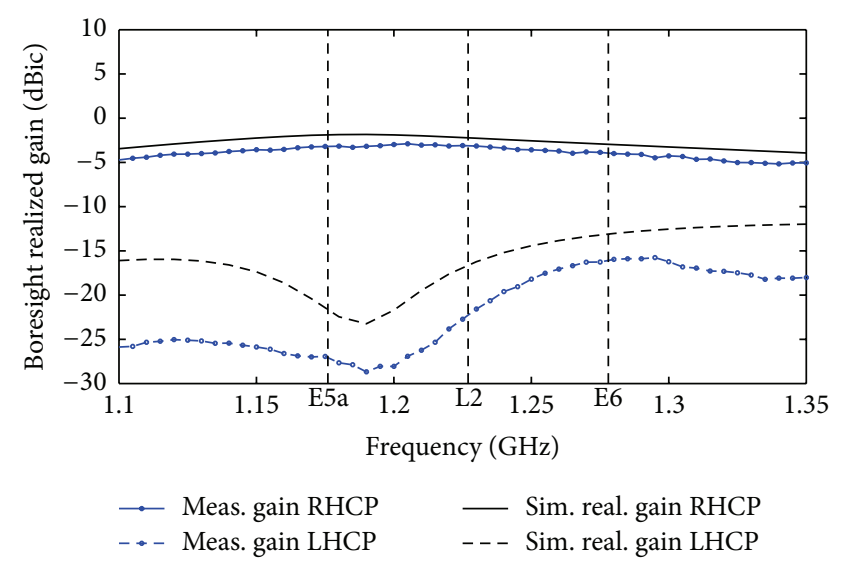

FIGURE 20: Boresight realized gain of the $2 \times 2$ DRA array with metallic fences and absorber versus frequency (one antenna fed).

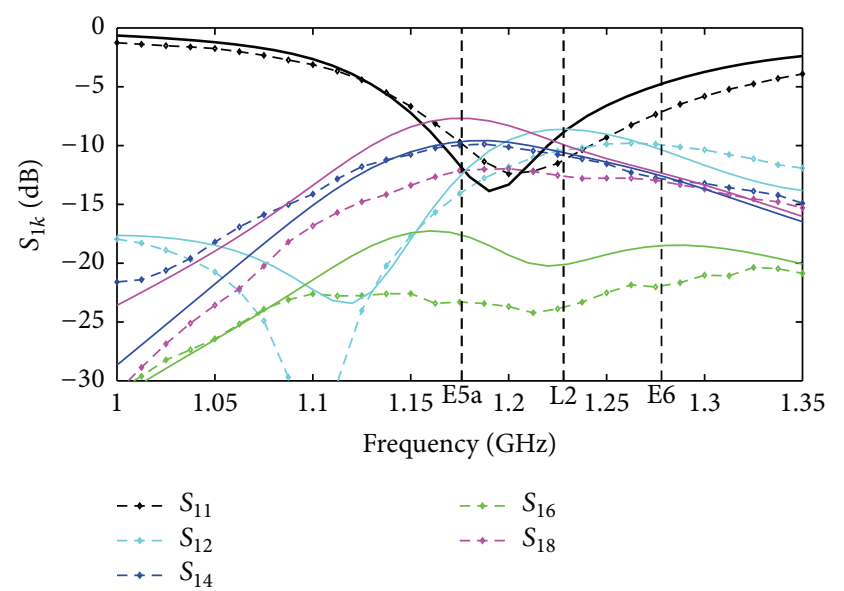

FIGURE 21: $S$-parameters (both simulated straight lines and measured dashed lines) of the $2 \times 2$ DRA array with metallic fences and absorber versus frequency (only the most relevant ones are shown for clarity's sake).

excitation between different antennas has been obtained with a commercial 1-to-4 sequential power combiner. As expected from simulation, a strong deterioration is experienced in embedded gain, due to mutual coupling effects. A relevant frequency shift is also clearly distinguishable, inhibiting the use of the array for the bands of interest.

4.3. $2 \times 2$ Array with Fences and Absorber. A cross-like metallic fence $(25 \mathrm{~mm}$ high and $1 \mathrm{~mm}$ thick) with $10 \mathrm{~mm}$ absorber ( 5 above and 5 below the edge) has then been added in-between the antennas in order to reduce mutual coupling, as shown in Section 3.2. The results of the measurements performed with the fence between the antennas are shown in Figures 20-22.

In this case, good agreement between simulation and measurement is found for both $S$-parameters and gain. For this configuration, optimal matching is obtained at $f_{0}=$ $1200 \mathrm{MHz}$, that is, in the middle of the bands of interest. A gain of $-3.2,-3.1$, and $-3.8 \mathrm{dBic}$ is achieved, respectively, at E5, L2, and E6 central frequencies. Though smaller than the 


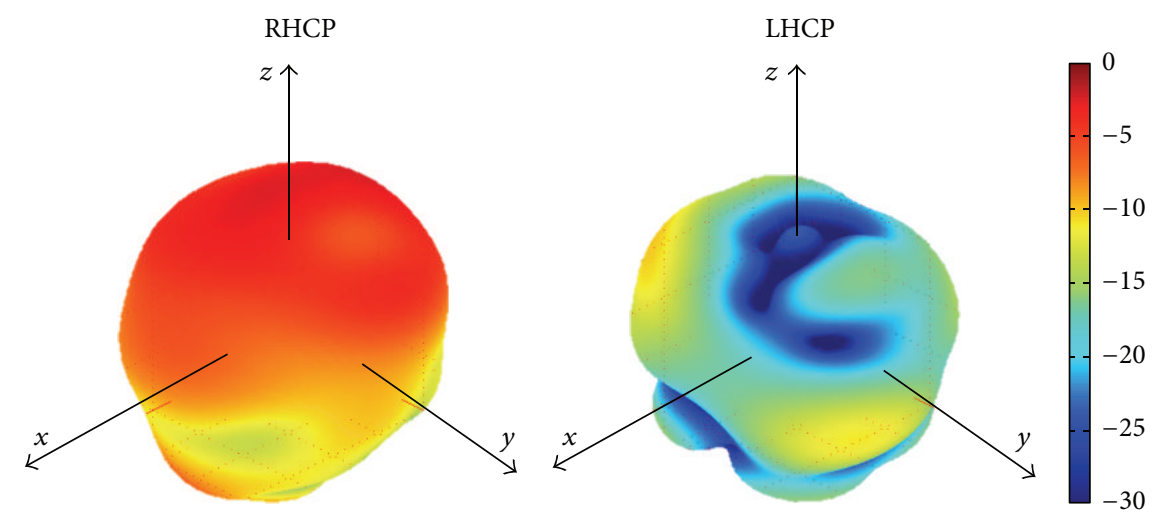

Figure 22: 3D embedded pattern for the RHCP and LHCP components of the $2 \times 2$ array with fences and absorber at $f_{0}=1200 \mathrm{MHz}$.

one of the single antenna configuration, the gain obtained in array configuration is still sufficient for successful satellite tracking and acquisition even when using only one output of the array: however, the use of multiple antennas in such a reduced footprint provides the additional capability of null steering and hence substantially improves robustness against interference with respect to the single antenna case. The 3D embedded pattern is moreover shown in Figure 22, with a clear indication of the almost hemispherical behaviour of the RHCP polarization and reduced cross polarization, hence enabling an optimal use of the array for precise and robust navigation purposes.

\section{Conclusions}

A miniaturized $2 \times 2$ array, made of dielectric resonator antennas, for robust GNSS navigation at E5/L2/E6 band has been shown. The use of a high DK material enables good miniaturization, helping to pack the $2 \times 2$ array in a footprint of $3.5^{\prime \prime}(=89 \mathrm{~mm})$ and hence making it suitable for mobile applications, for instance, in airplanes or cars. Metallic fences and a planar absorber are used to decrease mutual coupling due to the close vicinity of the antennas, hence managing to obtain strong cross polarization discrimination and reduced cross talk between the antennas and therefore promising optimal behaviour as miniaturized array for interference suppression in robust navigation applications.

\section{Competing Interests}

The authors declare that they have no competing interests.

\section{Acknowledgments}

The authors wish to thank Dr. M. Letz from Schott ag for valuable support with the dielectric material, Mr. L. Mair from Telemeter Electronic $\mathrm{GmbH}$ for providing the absorber, and B. Gabler for his help in measurement.

\section{References}

[1] D. Borio, C. O'Driscoll, and J. Fortuny, "Jammer impact on Galileo and GPS receivers," in Proceedings of the International
Conference on Localization and GNSS (ICL-GNSS '13), pp. 1-6, IEEE, Turin, Italy, June 2013.

[2] J. A. Larcom and H. Liu, "Modeling and characterization of GPS spoofing," in Proceedings of the 13th IEEE International Conference on Technologies for Homeland Security (HST '13), pp. 729-734, Waltham, Mass, USA, November 2013.

[3] M. V. T. Heckler, M. Cuntz, A. Konovaltsev, L. A. Greda, A. Dreher, and M. Meurer, "Development of robust safety-of-life navigation receivers," IEEE Transactions on Microwave Theory and Techniques, vol. 59, no. 4, pp. 998-1005, 2011.

[4] ARINC 743 Specification, http://www.aviation-ia.com.

[5] C. Fernández-Prades, L. L. Presti, and E. Falletti, "Satellite radiolocalization from GPS to GNSS and beyond: novel technologies and applications for civil mass market," Proceedings of the IEEE, vol. 99, no. 11, pp. 1882-1904, 2011.

[6] A. Konovaltsev, D. S. De Lorenzo, A. Hornbostel, and P. Enge, "Mitigation fo continuous and pulsed radio interference with GNSS antenna arrays," in Proceedings of the 21st International Technical Meeting of the Satellite Division of The Institute of Navigation (ION GNSS '08), Savannah, Ga, USA, September 2008 .

[7] M. G. Amin and W. Sun, "A novel interference suppression scheme for global navigation satellite systems using antenna array," IEEE Journal on Selected Areas in Communications, vol. 23, no. 5, pp. 999-1012, 2005.

[8] R. L. Fante and J. J. Vaccaro, "Wideband cancellation of interference in a GPS receive array," IEEE Transactions on Aerospace and Electronic Systems, vol. 36, no. 2, pp. 549-564, 2000.

[9] I. J. Gupta, T.-H. Iee, K. A. Griffith et al., "Non-planar adaptive antenna arrays for GPS receivers," IEEE Antennas and Propagation Magazine, vol. 52, no. 5, pp. 35-51, 2010.

[10] J. R. Lambert, C. A. Balanis, and D. DeCarlo, "Spherical cap adaptive antennas for GPS," IEEE Transactions on Antennas and Propagation, vol. 57, no. 2, pp. 406-413, 2009.

[11] K. Yinusa, W. Elmarissi, S. Caizzone, and A. Dreher, "A multiband conformal antenna array for GNSS applications," in Proceedings of the IEEE Antennas and Propagation Symposium (APS '16), Fajardo, Puerto Rico, USA, June-July 2016.

[12] N. Basta, A. Dreher, S. Caizzone et al., "System concept of a compact multi-antenna GNSS receiver," in Proceedings of the 7th German Microwave Conference (GeMiC'12), Ilmenau, Germany, March 2012. 
[13] M. Chen and C.-C. Chen, "A compact dual-band GPS antenna design," IEEE Antennas and Wireless Propagation Letters, vol. 12, pp. 245-248, 2013.

[14] J. Volakis, C.-C. Chen, and K. Fujimoto, Small Antennas: Miniaturization Techniques and Applications, McGraw-Hill, 2010.

[15] X. Ren, X. Chen, and K. Huang, "A novel electrically small meandered line antenna with a trident-shaped feeding strip for wireless applications," International Journal of Antennas and Propagation, vol. 2012, Article ID 757429, 7 pages, 2012.

[16] M. S. Alam, N. Misran, B. Yatim, and M. T. Islam, "Development of electromagnetic band gap structures in the perspective of microstrip antenna design," International Journal of Antennas and Propagation, vol. 2013, Article ID 507158, 22 pages, 2013.

[17] A. Sharma and S. C. Shrivastava, "Analysis of resonant frequency and quality factor of dielectric resonator at different dielectric constant materials," in Proceedings of the International Conference of Recent Advances in Microwave Theory and Applications (MICROWAVE '08), pp. 593-595, IEEE, Jaipur, India, November 2008.

[18] A. Petosa and A. Ittipiboon, "Dielectric resonator antennas: a historical review and the current state of the art," IEEE Antennas and Propagation Magazine, vol. 52, no. 5, pp. 91-116, 2010.

[19] Q. Lai, G. Almpanis, C. Fumeaux, H. Benedickter, and R. Vahldieck, "Comparison of the radiation efficiency for the dielectric resonator antenna and the microstrip antenna at $\mathrm{Ka}$ band," IEEE Transactions on Antennas and Propagation, vol. 56, no. 11, pp. 3589-3592, 2008.

[20] D. Mitra, S. M. Haque, and B. Ghosh, "Metal loaded miniaturized CPW fed DRA," in Proceedings of the Applied Electromagnetics Conference (AEMC '09), Kolkata, India, December 2009.

[21] Z.-N. Chen, K.-W. Leung, K.-M. Luk, and E. K.-N. Yung, "Effect of parasitic disk on a coaxial probe-fed dielectric resonator antenna," Microwave and Optical Technology Letters, vol. 15, no. 3, pp. 166-168, 1997.

[22] S. Caizzone, "Miniaturized E5a/E1 antenna array for robust GNSS navigation," IEEE Antennas and Wireless Propagation Letters, 2016.

[23] S. Caizzone and A. Dreher, "Miniaturized DRA array for GNSS applications," in Proceedings of the 9th European Conference on Antennas and Propagation (EuCAP '15), pp. 1-2, Lisbon, Portugal, May 2015.

[24] K. M. Pesyna, R. W. Heath, and T. E. Humphreys, "Centimeter positioning with a smartphone-quality GNSS antenna," in Proceedings of the 27th International Technical Meeting of the Satellite Division of the Institute of Navigation (ION GNSS '14), Tampa, Fla, USA, September 2014. 


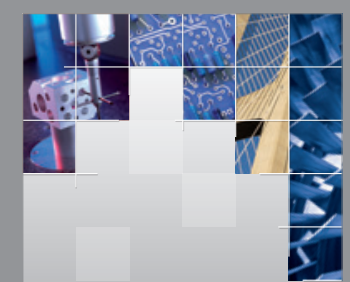

\section{Enfincering}
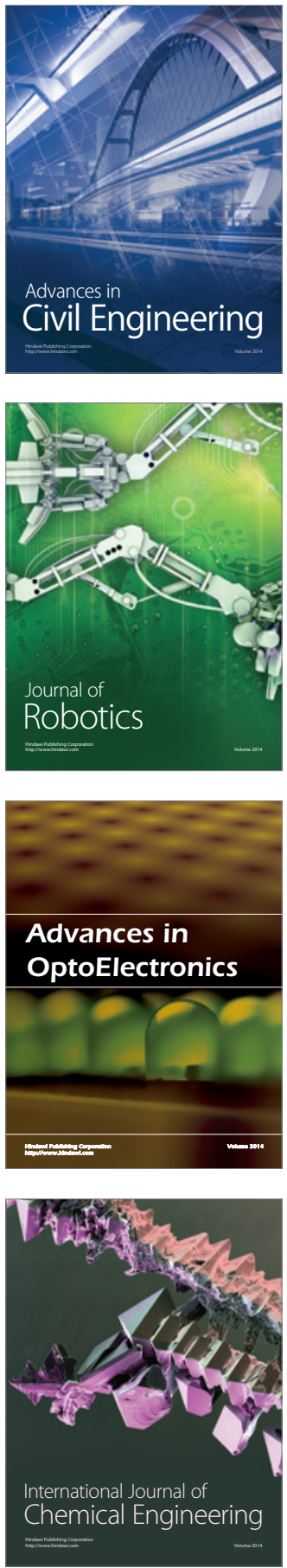

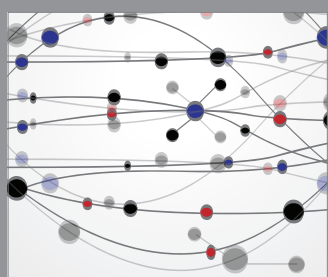

The Scientific World Journal

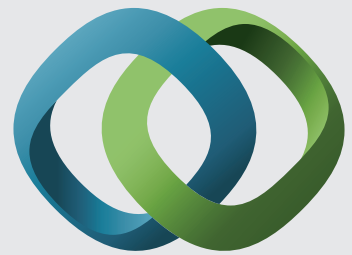

\section{Hindawi}

Submit your manuscripts at

http://www.hindawi.com
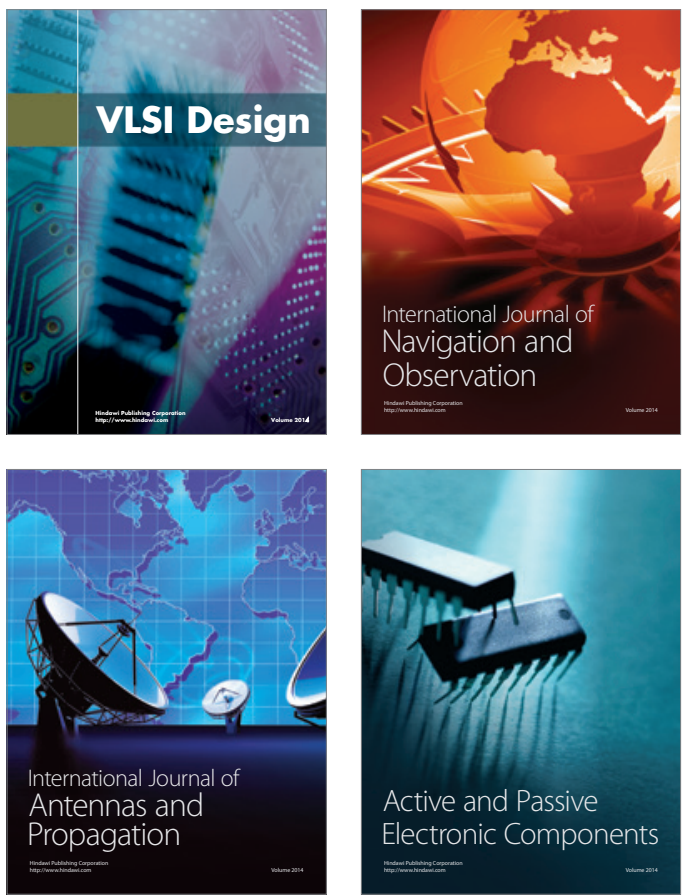
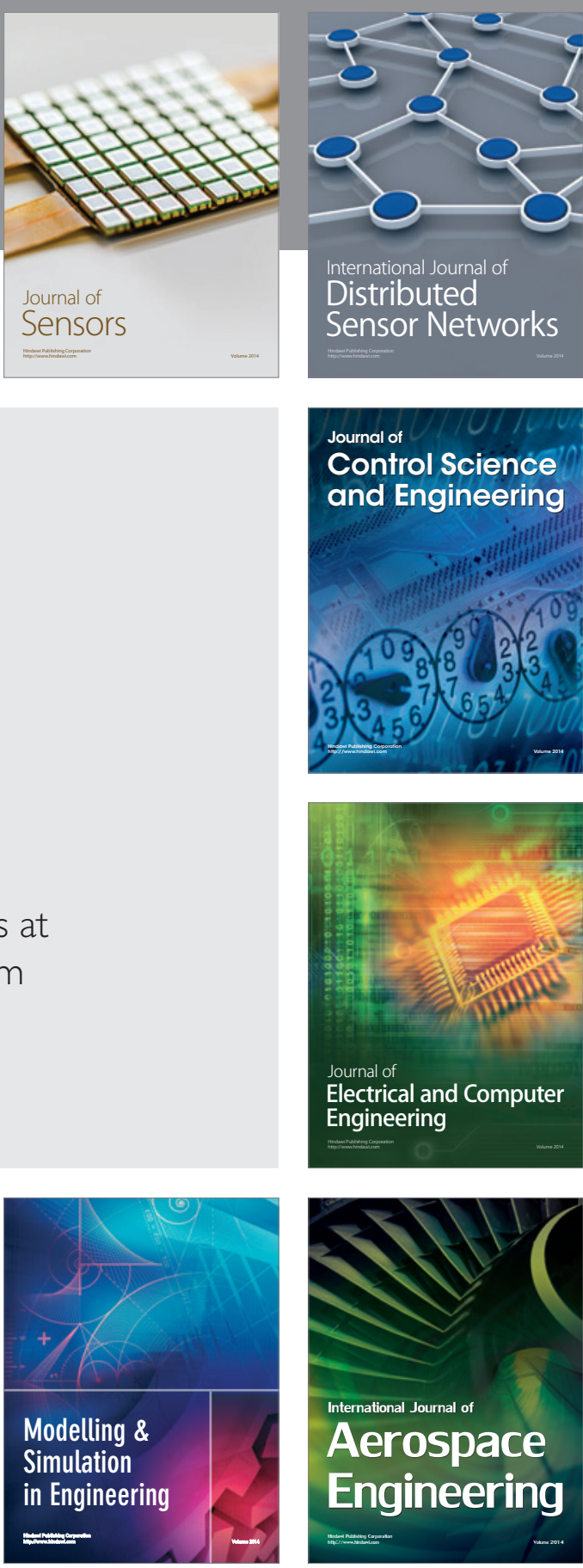

International Journal of

Distributed

Sensor Networks

Journal of

Control Science

and Engineering
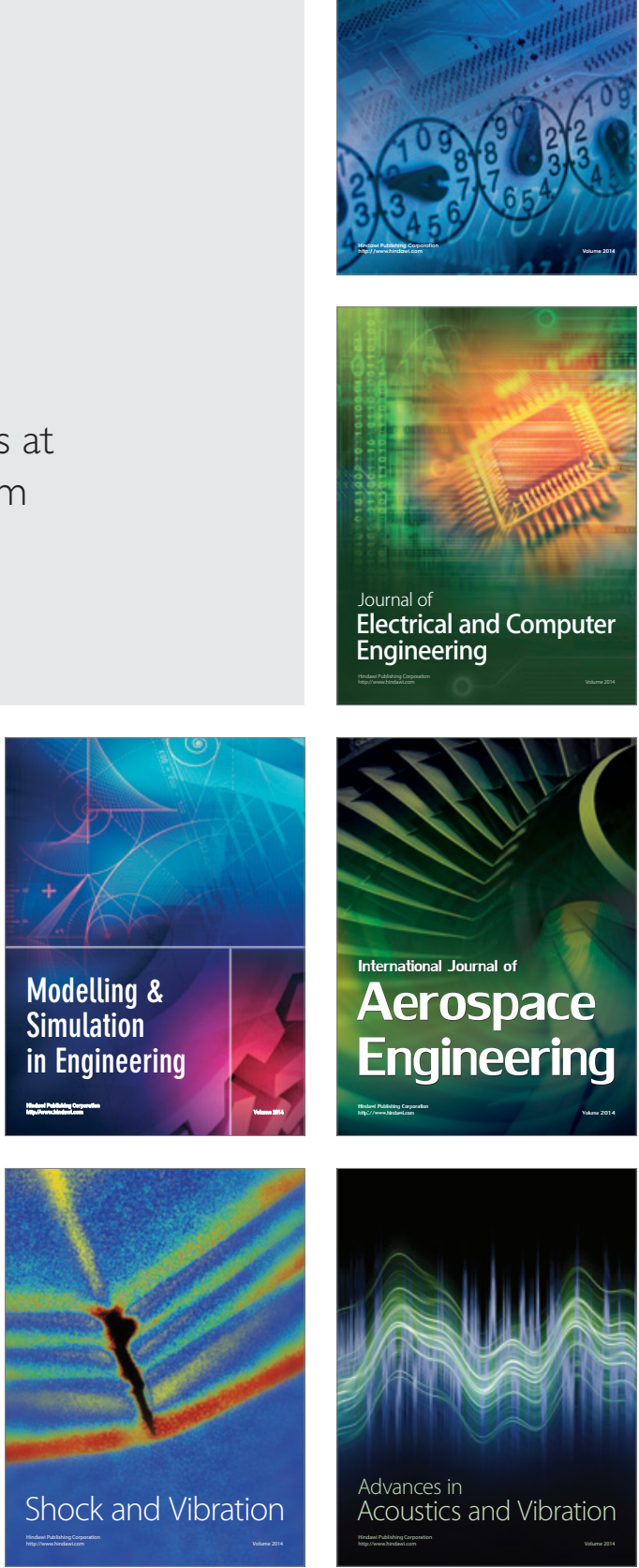